\title{
Partial-mixed special finite element for the analysis of multilayer composites and FGM
}

\author{
Orlando Andrianarison ${ }^{\mathrm{a} *}$ and Ayech Benjeddou ${ }^{\mathrm{b}}$ \\ ${ }^{a}$ Institut Supérieur de l'Automobile et des Transports, DRIVE Rue Mademoiselle Bourgeois, F-58660 \\ Nevers, France; ${ }^{b}$ Institut Supérieur de Mécanique de Paris, Innovations \& Structures 3 rue Fernand \\ Hainaut, F-93407 Saint Ouen cedex, France
}

\begin{abstract}
A partial-mixed special finite element (FE) is proposed for the static analysis of multilayer composite and functionally graded material plates. Using the Hamiltonian formalism, the three-dimensional elasticity equations are first reformulated so that a partial-mixed variational formulation, retaining as primary variables the translational displacements augmented with the transverse stresses only, is obtained; this allows, in particular, a straightforward fulfilment of the multilayer interfaces continuity conditions. After an only in-plane FE discretisation, the static problem is then reduced, for a single layer, to a Hamiltonian eigenvalue problem that is solved analytically, through the layer thickness, using the symplectic formalism; the multilayer solution is finally reached via the state-space method and the propagator matrix concept. The performance, in convergence and accuracy, of the proposed approach, applied to representative examples, is shown to be very satisfactory.
\end{abstract}

Un élément fini (EF) spécial partiellement mixte est proposé pour l'analyse des plaques en composite multicouche et matériau fonctionnellement gradué. Usant du formalisme Hamiltonien, les équations de l'élasticité tridimensionnelle sont d'abord reformulées telle qu'une formulation variationnelle, retenant comme variables indépendantes les déplacements de translation augmentés des contraintes transverses seulement, est obtenue; cela permet, en particulier, une satisfaction directe des conditions de continuité aux interfaces du multicouche. Après seulement une approximation plane par EF, le problème statique est ensuite réduit, pour une seule couche, à un problème aux valeurs propres Hamiltonien qui est résolu analytiquement, dans l'épaisseur de la couche, en utilisant le formalisme symplectique; finalement, la solution multicouche est atteinte via la matrice de propagation de la méthode d'espace d'état. La performance, en convergence et précision, de l'approche proposée, appliquée à des exemples représentatifs, est montrée très satisfaisante.

Keywords: Hamiltonian approach; semi-analytical solution; partial-mixed finite element; state space method; composite; functionally graded material; plate.

Mots-Clés: Approche Hamiltonienne; solution semi-analytique; élément fini partiellement mixte; méthode d'espace d'état; composite; matériau fonctionnellement gradué; plaque.

\section{Introduction}

Since the late 1970s, composite materials have grown rapidly in almost all industries, in particular for aeronautics, where they were early reserved to secondary structural components;

*Corresponding author. Email: orlando.andrianarison@u-bourgogne.fr 
but, their recent success for jumbo airliners construction helped them to be planned currently for load-carrying main structural elements, like fuselage and wings. They are searched, in particular, for their undeniable advantages, like lightweight, resistance, isolation. Nevertheless, they are also known to have severe disadvantages like sensitivity to impact and vibration that may lead to their fatigue, and damage, mainly in the form of delamination, wrinkling. Often, the latter localised phenomena analysis requires three-dimensional (3D) modelling tools in order to predict correctly their 3D stress state. Also, for aeronautic composites, which are often made of stacked uni-directional laminae, the transverse stresses continuity at the interfaces should be fulfilled, and accurate stresses evaluation, for damage detection for example, should be reached.

Functionally graded materials (FGM) form another class of nowadays advanced materials that find wide research interest during the last decades (Birman \& Byrd, 2007). They are mainly searched for minimising the concentration of the interface stresses in multilayer composites. Even for a single-layer FGM, the grading property, often considered along the structural component thickness, can be simply modelled using numerical (virtual) layers stacking so that the FGM is seen as an equivalent laminate. Hence, its multilayer interfaces stresses have to be continuous and accurately predicted in order to optimise its thickness grading for example.

In summary, modelling and analyses of composites and FGM need to represent their layerwise aspect, fulfil their transverse stresses interface continuities (IC) and predict accurately their 3D stress state. Among the numerous available models (Kreja, 2011; Zhang \& Yang, 2009), only a few can cope with these specific features. Numerically, hybrid and full mixed finite elements (FE) (Brezzi \& Fortin, 1991) can be used but remain expensive. Analytically, the partial-mixed state-space method (SSM) (Bahar, 1977), that uses the displacements and transverse stresses as independent variables, is an alternative but is limited by the a priori fulfilment of the boundary conditions (BC). A third option is the so-called semi-analytical approach that combines analytical SSM and in-plane or thickness numerical approximation.

Partial-mixed variational formulations (VF) are well suited for modelling multilayer composites and FGM since they augment the translational displacements with the transverse stresses only as independent variables (Benjeddou \& Andrianarison, 2006; Robaldo, Benjeddou, \& Carrera, 2005); these three-field partial-mixed VF can be proved using partial Legendre transformation (Reissner, 1984) or Lagrange multipliers (Benjeddou \& Andrianarison, 2006). The present work will show later that this can be also proved using the Hamiltonian formalism which is well known in classical mechanics (Goldstein, Poole, \& Safko, 2002). Partial mixed VF has the feature to naturally fulfil the laminate transverse stresses IC without cumbersome manipulations as for equivalent single-layer or layer-wise classical two-dimensional (2D) theories (Kreja, 2011).

Semi-analytical approaches have been proposed for the analysis of composite structures since the early 1990s (Steele \& Kim, 1992). They differ by the numerical approximation used through the laminate thickness or plane, and the evaluation method of the state matrix exponential. Hence, Zou and Tang (1995) used in-plane partial-mixed FE approximation and a power series expansion; Sheng and Ye (2002) retained full mixed FE and precise time (substituted by the thickness coordinate) step integration method, while they used later (Sheng \& Ye, 2005) discrete-layer FE and eigenvalue solution. Few contributions exist also on the use of semi-analytical solutions for the analysis of 3D FGM (Wu \& Li, 2010a) and 2D elastic (Li, Zhong, \& Tian, 2011) plates.

The reformulation of the equations of 3D (Steele \& Kim, 1992; Zou \& Tang, 1995) or 2D (Li, Zhong, \& Tian, 2011) elasticity in the Hamiltonian framework allows introducing naturally the transverse stresses as primary unknowns thanks to a Legendre transformation. 
This makes the final partial differential equations (PDE) lower in order compared to classical displacement formulations. Namely, as it will be shown later, the Hamiltonian formalism allows transforming in a systematic way the equations of elasticity (fourth-order PDE) into first-order linear ordinary differential equations (ODE) where the coordinate in the thickness direction is the only independent variable. This feature is quite interesting in the perspective of developing numerical tools for multilayered plates since the propagator matrix concept, used for analytical methods (Bahar, 1977), can then be used. However, the first-order state space equation, resulting from the Hamiltonian system, leads automatically to a state matrix exponential that is solved using either the power series expansion of the exponential, as in Zou and Tang (1995) for 3D plates, or the symplectic approach, as in Li, Zhong, and Tian (2011) for 2D ones.

From the above literature analysis, it appears that the investigation of the multilayer composites and FGM response using a three-field partial-mixed VF resulting from the Hamiltonian formalism and the associated exponential system matrix resolution using the symplectic approach is a suitable choice and remains an original semi-analytical methodology. This is reached by combining an in-plane $\mathrm{FE}$ discretisation of the here retained plate structure and the SSM-based analytical solution through the thickness; the size of the problem to be solved is then independent of the number of layers of the composite, and no interpolation along the thickness is needed thanks to the exact integration along this transverse direction. Also, the here adopted approach makes interlaminar stresses continuities straightforward thanks to the propagator matrix formalism which provides a simple and systematic way to transmit the state variables from the bottom to the top of the laminate. Claimed originalities of the present work include Hamiltonian-based derivation of the partial-mixed VF and the associated symplectic solution for multilayer composites and FGM.

In the following, starting from a Lagrange VF, the Hamiltonian one will be first derived. Then, the semi-analytical approach, that combines in-plane FE discretisation and thickness symplectic - SSM solution, is given. Finally, for its performance assessment, the proposed special FE is applied to selected benchmarks.

\section{Hamiltonian variational formulation}

The considered multilayer plate structure, of 3D body $\Omega$ to which a Cartesian global coordinate system $(O, x, y, z)$ is attached, is shown in Figure 1 . It is considered body force - free but subjected to a surface traction vector $\underline{\mathbf{F}}$ on the part $\Gamma_{F}$ of its regular boundary $\delta \Omega=\Gamma$ and a displacement vector $\underline{\mathbf{u}}$ (not shown) on its complementary part $\Gamma_{u}$ so that $\Gamma_{u} \cup \Gamma_{F}=\Gamma$ and $\Gamma_{u} \cap \Gamma_{F}=\varnothing$. Simple underlined quantities denote vectors and double underlined ones denote matrices.

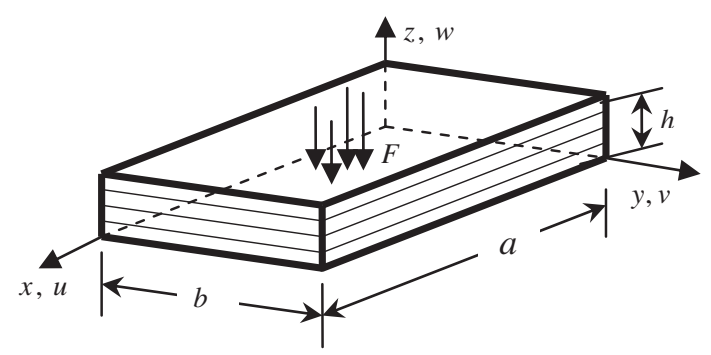

Figure 1. Multilayer plate geometry and notations. 
The following Lagrange functional defines the total potential energy associated to the above described linear elastic body:

$$
L(\underline{\mathrm{u}})=\int_{\Omega} E(\underline{\mathrm{u}}) d \Omega-\int_{\Gamma_{F}} \underline{\mathrm{u}}^{T} \underline{\mathrm{F}} d \Gamma
$$

With the internal elastic energy density having this form:

$$
E(\underline{\mathbf{u}})=\frac{1}{2^{\underline{\varepsilon}}} \underline{\underline{\mathbf{C}}} \underline{\boldsymbol{\varepsilon}}
$$

In which, $\underline{\varepsilon}$ is the engineering linear strains vector and $\underline{\mathbf{C}}$ is the elasticity matrix.

The static equilibrium of the elastic body is then obtained from the principle of minimum total potential energy which leads to:

$$
\delta \int_{\Omega} E(\underline{\mathbf{u}}) d \Omega-\int_{\Gamma_{F}} \delta \underline{\mathbf{u}}^{T} \underline{\mathbf{F}} d \Gamma=0
$$

where, the displacement vector $\underline{\mathbf{u}}$ is searched as kinematically admissible, and its variation $\delta \underline{\mathbf{u}}$ is supposed to be regular and nil on $\Gamma_{u}$.

In the case of a layered body, the adjoining laminae are presumed to be perfectly bonded together so that the displacements and transverse stresses should be continuous through their interfaces. As discussed in the introduction, the satisfaction of the IC is a crucial point in the development of reliable multilayered structures models. This is tackled here via the Hamiltonian formalism. For this purpose, the 3D strain-displacement relations are split into transverse $(t)$ and in-plane $(p)$ contributions as follows:

$$
\underline{\boldsymbol{\varepsilon}}_{t}=\underline{\dot{\mathbf{u}}}+\underline{\underline{\mathbf{D}}}_{1} \underline{\mathbf{u}}, \underline{\boldsymbol{\varepsilon}}_{p}=\underline{\underline{\mathbf{D}}}_{2} \underline{\mathbf{u}}
$$

where,

$$
\underline{\mathbf{u}}=\frac{\partial u}{\partial z}, \underline{\underline{\mathbf{D}}}_{1}=\left[\begin{array}{ccc}
0 & 0 & \partial_{x} \\
0 & 0 & \partial_{y} \\
0 & 0 & 0
\end{array}\right], \quad \underline{\underline{\mathbf{D}}}_{2}=\left[\begin{array}{ccc}
\partial_{x} & 0 & 0 \\
0 & \partial_{y} & 0 \\
\partial_{y} & \partial_{x} & 0
\end{array}\right]
$$

$\partial_{x}\left(\right.$ resp. $\left.\partial_{y}\right)$ is a partial derivation with respect to $x$ (resp. $y$ ) coordinate, and the upper dot c., denoting a partial derivation with respect to the thickness coordinate $z$ is a classical mechanics (Goldstein, Poole, \& Safko, 2002) standard notation practise which makes use of an analogy with time derivation in classical Hamiltonian formalism.

Above splitting of the strain vector into in-plane and normal contributions leads to the following similar decomposition of the linear elastic constitutive equations:

$$
\left\{\begin{array}{l}
\underline{\sigma}_{p} \\
\underline{\sigma}_{t}
\end{array}\right\}=\left[\begin{array}{ll}
\underline{\underline{C}}_{p p} & \underline{\underline{C}}_{p t} \\
\underline{\underline{C}}_{p t}^{T} & \underline{\underline{C}}_{t t}
\end{array}\right]\left\{\begin{array}{l}
\underline{\varepsilon}_{p} \\
\underline{\varepsilon}_{t}
\end{array}\right\}
$$

where, $\underline{\boldsymbol{\sigma}}_{p}=\left\{\boldsymbol{\sigma}_{x x}, \boldsymbol{\sigma}_{y y}, \boldsymbol{\sigma}_{x y}\right\}^{T}$ and $\underline{\boldsymbol{\sigma}}_{t}=\left\{\boldsymbol{\sigma}_{x z}, \boldsymbol{\sigma}_{y z}, \boldsymbol{\sigma}_{z z}\right\}^{T}$ refer to in-plane and transverse stress vector contributions. The strain vector ones are similarly defined, and the elastic bloc matrices are in Benjeddou and Andrianarison (2006), Robaldo, Benjeddou, and Carrera (2005).

Substituting Equations (4) and (5) in (2), transforms Equation (3) into: 


$$
\delta \int_{\Omega} E(\underline{\mathbf{u}}, \underline{\mathbf{u}}) d \Omega-\int_{\Gamma_{F}} \delta \underline{\mathbf{u}}^{T} \underline{\mathbf{F}} d \Gamma=0
$$

where,

$$
E(\underline{\mathbf{u}}, \underline{\dot{\mathbf{u}}})=\frac{1}{2^{\underline{\boldsymbol{\varepsilon}_{p}}}} \underline{\underline{\mathbf{C}}}_{p p} \underline{\boldsymbol{\varepsilon}_{p}}+\underline{\boldsymbol{\varepsilon}}_{t}^{T} \underline{\underline{\mathbf{C}}}_{p t}^{T} \boldsymbol{\varepsilon}_{p}+\frac{1}{2^{\boldsymbol{\varepsilon}_{t}^{T}}} \underline{\underline{\mathbf{C}}}_{t} \boldsymbol{\varepsilon}_{t}
$$

The construction of the Hamiltonian variational equation, corresponding to Equation (3), requires the determination of the dual, or conjugate, variable to $\underline{\mathbf{i}}$ (i.e. the generalised momentum $\underline{\mathbf{p}}$ ); this is achieved from Equations (4), (7) as follows:

$$
\underline{\mathbf{p}}=\frac{\partial E}{\partial \underline{\dot{\mathbf{u}}}}=\frac{\partial E}{\partial \underline{\boldsymbol{\varepsilon}}_{t}} \frac{\partial \underline{\boldsymbol{\varepsilon}}_{t}}{\partial \underline{\mathbf{u}}}=\underline{\underline{\mathbf{C}}}_{t t} \underline{\boldsymbol{\varepsilon}}_{t}+\underline{\underline{\mathbf{C}}}_{p t}^{T} \underline{\boldsymbol{\varepsilon}}_{p}=\underline{\boldsymbol{\sigma}}_{t}
$$

It can then be seen that the conjugate variable $\mathbf{p}$, relative to the displacement thickness-gradient vector $\underline{\dot{\mathbf{u}}}$, coincides with the normal stress one $\underline{\boldsymbol{\sigma}}_{t}$. Substitution of Equation (4) in Equation (8) thus leads to the following relation:

$$
\underline{\boldsymbol{\sigma}}_{t}=\underline{\underline{\mathbf{C}}}_{t} \underline{\dot{\mathbf{u}}}+\underline{\underline{\mathbf{C}}}_{t} \underline{\underline{\mathbf{D}}}_{1} \underline{\mathbf{u}}+\underline{\underline{\mathbf{C}}}_{p t}^{T} \underline{\underline{\mathbf{D}}}_{2} \underline{\mathbf{u}}
$$

From which this thickness-gradient displacement expression can be deduced:

$$
\underline{\dot{\mathbf{u}}}=\underline{\underline{\mathbf{C}}}_{t t}^{-1} \underline{\boldsymbol{\sigma}}_{t}-\underline{\underline{\mathbf{D}}}_{1} \underline{\mathbf{u}}-\underline{\underline{\mathbf{C}}}_{t t}^{-1} \underline{\mathbf{C}}_{p t}^{T} \underline{\underline{\mathbf{D}}}_{2} \underline{\mathbf{u}}
$$

Elimination of $\underline{\mathbf{u}}$ from Equations (6) and (7), with the help of Equation (10), then transforms Equation (1) into:

$$
L\left(\underline{\mathbf{u}}, \underline{\boldsymbol{\sigma}}_{t}\right)=\int_{\Omega} E\left(\underline{\mathbf{u}}, \underline{\boldsymbol{\sigma}}_{t}\right) d \Omega-\int_{\Gamma_{F}} \underline{\mathbf{u}}^{T} \underline{\mathbf{F}} d \Gamma
$$

where,

$$
E\left(\underline{\mathbf{u}}, \underline{\boldsymbol{\sigma}}_{t}\right)=\frac{1}{2} \underline{\mathbf{u}}^{T} \underline{\underline{\mathbf{D}}}_{2}^{T}\left(\underline{\underline{\mathbf{C}}}_{p p}-\underline{\underline{\mathbf{C}}}_{p t} \underline{\underline{\mathbf{C}}}_{t t}^{-1} \underline{\underline{\mathbf{C}}}_{p t}^{T}\right) \underline{\underline{\mathbf{D}}}_{2} \underline{\mathbf{u}}+\frac{1}{2} \underline{\boldsymbol{\sigma}}_{t}^{T} \underline{\underline{\mathbf{C}}}_{t t}^{-1} \underline{\boldsymbol{\sigma}}_{t}
$$

The Hamiltonian energy density is now introduced via this Legendre transform (Goldstein, Poole, \& Safko, 2002) of Equation (11):

$$
h\left(\underline{\mathbf{u}}, \underline{\boldsymbol{\sigma}}_{t}\right)=\underline{\boldsymbol{\sigma}}_{t}^{T} \underline{\mathbf{u}}-E\left(\underline{\mathbf{u}}, \underline{\boldsymbol{\sigma}}_{t}\right)
$$

where $\underline{\mathbf{u}}$ is here also expressed in terms of $\left(\underline{\mathbf{u}}, \underline{\boldsymbol{\sigma}_{t}}\right)$ with the help of Equation (10). Equation (13) leads then to this Hamiltonian functional:

$$
H\left(\underline{\mathbf{u}}, \underline{\boldsymbol{\sigma}}_{t}\right)=\int_{\Omega}\left[\underline{\boldsymbol{\sigma}}_{t}^{T} \underline{\mathbf{u}}-h\left(\underline{\mathbf{u}}, \underline{\boldsymbol{\sigma}}_{t}\right)\right] d \Omega-\int_{\Gamma_{F}} \underline{\mathbf{u}}^{T} \underline{\mathbf{F}} d \Gamma
$$

Taking the variation of Equation (14) and applying Green's formula provides:

$$
\int_{\Omega}\left[\delta \underline{\boldsymbol{\sigma}}_{t}^{T} \underline{\mathbf{u}}-\delta \underline{\mathbf{u}}^{T} \underline{\dot{\sigma}}_{t}-\left(\frac{\partial h}{\partial \underline{\mathbf{u}}^{2}}\right)^{T} \delta \underline{\mathbf{u}}-\left(\frac{\partial h}{\partial \underline{\boldsymbol{\sigma}}_{t}}\right)^{T} \delta \underline{\boldsymbol{\sigma}}_{t}\right] d \Omega+\int_{\partial \Omega} \delta \underline{\mathbf{u}}^{T} \underline{\boldsymbol{\sigma}}_{t} d \Gamma-\int_{\Gamma_{F}} \delta \underline{\mathbf{u}^{T}} \underline{\mathbf{F}} d \Gamma=0
$$


Then, grouping together the terms relative to the same virtual variable, the previous equation turns into:

$$
\int_{\Omega} \delta \underline{\mathbf{u}}^{T}\left(-\underline{\dot{\sigma}}_{t}-\frac{\partial h}{\partial \underline{\mathbf{u}}}\right) d \Omega+\int_{\Omega} \delta \underline{\boldsymbol{\sigma}}_{t}^{T}\left(\underline{\mathbf{u}}-\frac{\partial h}{\partial \underline{\boldsymbol{\sigma}}_{t}}\right) d \Omega+\int_{\Gamma_{F}} \delta \underline{\mathbf{u}}^{T}\left(\underline{\boldsymbol{\sigma}}_{t}-\underline{\mathbf{F}}\right) d \Gamma=0, \forall \delta \underline{\mathbf{u}} / \delta \underline{\mathbf{u}}=0\left(\Gamma_{u}\right)
$$

Using Equation (13) in Equation (16), the Hamiltonian variational formulation finally writes in the following form:

$$
\begin{gathered}
\int_{\Omega} \delta \underline{\mathbf{u}}^{T}\left(-\underline{\dot{\boldsymbol{\sigma}}}_{t}+\underline{\underline{\mathbf{D}}}_{2}^{T} \underline{\underline{\mathbf{C}}}_{p p}^{*} \underline{\underline{\mathbf{D}}}_{2} \underline{\mathbf{u}}+\underline{\underline{\mathbf{D}}}_{1}^{T} \underline{\boldsymbol{\sigma}}_{t}+\underline{\underline{\mathbf{D}}}_{2}^{T} \underline{\mathbf{C}}_{p t}^{*} \underline{\boldsymbol{\sigma}}_{t}\right) d \Omega \\
\quad+\int_{\Omega} \delta \underline{\boldsymbol{\sigma}}_{t}^{T}\left(\underline{\mathbf{u}}^{-} \underline{\mathbf{C}}_{t t}^{*} \underline{\boldsymbol{\sigma}}_{t}+\underline{\underline{\mathbf{D}}}_{1} \underline{\mathbf{u}}^{+}+\underline{\mathbf{C}}_{p t}^{* T} \underline{\underline{\mathbf{D}}}_{2} \underline{\mathbf{u}}\right) d \Omega=0 \\
\forall \underline{\mathbf{u}} / \underline{\mathbf{u}}=\underline{\overline{\mathbf{u}}}\left(\Gamma_{u}\right) ; \quad \forall \underline{\boldsymbol{\sigma}}_{t} / \underline{\boldsymbol{\sigma}}_{t}=\underline{\mathbf{F}}\left(\Gamma_{F}\right) \\
\forall \underline{\mathbf{u}} / \delta \underline{\mathbf{u}}=\underline{\mathbf{0}}\left(\Gamma_{u}\right) ; \quad \forall \delta \underline{\boldsymbol{\sigma}}_{t} / \delta \underline{\boldsymbol{\sigma}}_{t}=\underline{\mathbf{0}}\left(\Gamma_{F}\right)
\end{gathered}
$$

In which the starred matrices are elastic ones, modifying those in (5), defined as:

$$
\underline{\underline{\mathbf{C}}}_{t t}^{*}=\underline{\underline{\mathbf{C}}}_{t t}^{-1}, \underline{\underline{\mathbf{C}}}_{p p}^{*}=\underline{\underline{\mathbf{C}}}_{p p}-\underline{\underline{\mathbf{C}}}_{p t} \underline{\underline{\mathbf{C}}}_{t t}^{-1} \underline{\underline{\mathbf{C}}}_{p t}^{T}, \underline{\underline{\mathbf{C}}}_{p t}^{*}=\underline{\underline{\mathbf{C}}}_{p t} \underline{\underline{\mathbf{C}}}_{t t}^{-1}
$$

\section{Partial-mixed special finite element - symplectic state-space solution}

In order to derive the general solution of the problem, let us consider, in a first subsection, the case of a single-layer plate, for which the FE in-plane discretisation of the Hamiltonian $\mathrm{VF}$ is introduced and the symplectic solution of the resulting state space first-order equation is presented. Then, in a second subsection, the previous single-layer solution is extended to the multilayer case via the SSM - propagator matrix recursively by exploiting the laminate IC and bottom and top stress BC.

\subsection{Finite element discretisation and symplectic single-layer solution}

The single-layer domain is decomposed into its in-plane area $A$, of range $[0, a] \times[0, b]$ and its thickness, of range $[0, h]$. Let's then suppose that the primary variables $\left(\underline{\mathbf{u}}, \underline{\boldsymbol{\sigma}}_{t}\right)$ at any point of the domain have the following separable discretisations:

$$
\underline{\mathbf{u}}(x, y, \zeta)=\underline{\underline{\mathbf{N}}}(x, y) \underline{\mathbf{U}}(\zeta) ; \quad \underline{\boldsymbol{\sigma}}_{t}(x, y, \zeta)=\underline{\underline{\mathbf{N}}}(x, y) \underline{\mathbf{\Sigma}}(\zeta)
$$

where, $\mathbf{N}(x, y)$ is the interpolation matrix containing the FE shape functions, and as shown in Figure $\overline{\overline{2}}, \zeta$ refers to the local coordinate relative to a given layer and $z$ denotes the plate global coordinate.

Substituting Equation (19) into the variational equation (17) leads to its following discretised version:

$$
\int_{0}^{h} \delta \underline{U^{T}}\left(-\underline{\underline{\mathbf{N}}} \underline{\underline{\boldsymbol{\Sigma}}}+\underline{\underline{\mathbf{B}}} \underline{\mathbf{U}}+\underline{\underline{\mathbf{A}}}^{T} \underline{\boldsymbol{\Sigma}}\right) d \zeta+\int_{0}^{h} \delta \underline{\underline{\boldsymbol{\Sigma}^{T}}}(\underline{\underline{\mathbf{N}}} \underline{\dot{\mathbf{U}}}+\underline{\underline{\tilde{A}}} \underline{U}-\underline{\underline{\mathbf{D}}} \underline{\boldsymbol{\Sigma}}) d \zeta=0
$$

where,

$$
\begin{aligned}
& \underline{\underline{\tilde{\mathbf{N}}}}=\int_{A} \underline{\underline{\mathbf{N}}}^{T} \underline{\underline{\mathbf{N}}} d A, \quad \underline{\underline{\tilde{\mathbf{B}}}}=\int_{A} \underline{\underline{\mathbf{N}}}^{T} \underline{\underline{\mathbf{D}}}_{2}^{T} \underline{\underline{\mathbf{C}}}_{p p}^{*} \underline{\underline{\mathbf{D}}}_{2} \underline{\underline{\underline{N}}} d A \\
& \underline{\underline{\mathbf{\mathbf { A }}}}=\int_{A} \underline{\underline{\underline{\mathbf{N}}}}^{T}\left(\underline{\underline{\mathbf{D}}}_{1}+\underline{\underline{\mathbf{C}}}_{p t}^{* T} \underline{\underline{\mathbf{D}}}_{2}\right) \underline{\underline{\mathbf{N}}} d A, \quad \underline{\underline{\mathbf{D}}}=\int_{A} \underline{\underline{\mathbf{N}}}^{T} \underline{\underline{\mathbf{C}}}_{t t}^{*} \underline{\underline{\mathbf{N}}} d A
\end{aligned}
$$




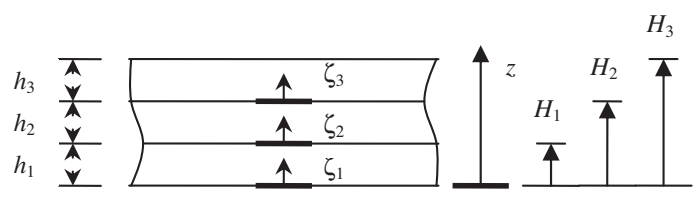

Figure 2. Illustration of global $(z)$ and local $(\zeta)$ coordinates.

From Equation (20), usual procedures of variational calculus (Courant \& Hilbert, 1989; Washizu, 1968) allow reaching this first-order state-space matrix equation:

$$
\left\{\begin{array}{c}
\underline{\dot{\mathrm{U}}} \\
\underline{\underline{\dot{\Sigma}}}
\end{array}\right\}=\left[\begin{array}{cc}
-\underline{\underline{\mathrm{A}}} & \underline{\underline{\mathrm{D}}} \\
\underline{\underline{\mathrm{B}}} & \underline{\underline{\mathrm{A}}}^{\mathrm{T}}
\end{array}\right]\left\{\begin{array}{l}
\underline{\mathrm{U}} \\
\underline{\Sigma}
\end{array}\right\}
$$

In which the bloc matrices are defined from those defined in Equation (21) by:

$$
\underline{\underline{\mathbf{A}}}=\underline{\underline{\mathbf{N}}}^{-1} \underline{\underline{\mathbf{A}}} ; \quad \underline{\underline{\mathbf{D}}}=\underline{\underline{\mathbf{N}}}^{-1} \underline{\underline{\mathbf{D}}} ; \quad \underline{\underline{\mathbf{B}}}=\underline{\underline{\tilde{\mathbf{N}}}}^{-1} \underline{\underline{\mathbf{\mathbf { B }}}}
$$

Introducing this extended vector, $\underline{\tilde{\boldsymbol{\Psi}}}^{T}=\{\underline{\mathbf{U}}, \underline{\boldsymbol{\Sigma}}\}^{T}$, Equation (22) can be rewritten in the following concise form:

$$
\underline{\dot{\boldsymbol{\Psi}}}(x, y, \zeta)=\underline{\underline{\mathbf{H}}}(x, y) \underline{\tilde{\mathbf{\Psi}}}(\zeta)
$$

with,

$$
\underline{\underline{\mathbf{H}}}(x, y)=\left[\begin{array}{cc}
-\underline{\underline{\mathbf{A}}} & \underline{\underline{\mathbf{D}}} \\
\underline{\underline{\mathbf{B}}} & \underline{\underline{\mathbf{A}}}
\end{array}\right]
$$

Being the Hamiltonian matrix $\underline{\underline{\mathbf{H}}}$ that contains all plane variables dependence; it has these symmetry properties:

$$
\underline{\underline{\mathbf{D}}}=\underline{\underline{\mathbf{D}}}^{\mathrm{T}} ; \quad \underline{\underline{\mathbf{B}}}=\underline{\underline{\mathbf{B}}}^{T}
$$

The solution of the first-order differential Equation (24) can be searched in this exponential form:

$$
\underline{\tilde{\boldsymbol{\Psi}}}=\underline{\boldsymbol{\Psi}} e^{\mu \zeta}
$$

Substituting then this last expression of $\underline{\tilde{\boldsymbol{\Psi}}}$ back into Equation (24) leads to the following eigenvalue problem:

$$
\underline{\underline{\mathbf{H}}} \underline{\boldsymbol{\Psi}}=\mu \underline{\boldsymbol{\Psi}}
$$

It is worth noting that Equation (27) is a Hamiltonian eigenvalue problem due to the Hamiltonian topology of the matrix $\underline{\underline{\mathbf{H}}}$ (Benner, Mehrmann \& Xu, 1998; Van Loan, 1984). One additional symmetry property specific to the Hamiltonian matrix is:

$$
(\underline{\underline{\mathbf{H}}} \underline{\underline{\mathbf{J}}})^{T}=\underline{\underline{\mathbf{H}}} \underline{\underline{\mathbf{J}}}
$$


with,

$$
\underline{\underline{\mathbf{J}}}=\left[\begin{array}{cc}
\mathbf{0} & \underline{\mathbf{I}}(n, n) \\
-\underline{\underline{\mathbf{I}}}(n, n) & \mathbf{0}
\end{array}\right]
$$

Being the so-called unit symplectic matrix of size $2 n$, and $\underline{\mathbf{I}}$ is the identity matrix of order $n$ corresponding to the number of degrees of freedom resulting from the FE discretisation; $\underline{\underline{\mathbf{J}}}$ obeys the following algebraic properties:

$$
\underline{\underline{\mathbf{J}}}^{2}=-\underline{\underline{\mathbf{I}}}(2 n, 2 n) ; \underline{\underline{\mathbf{J}}}^{-1}=\underline{\underline{\mathbf{J}}}^{T}
$$

Due to the Hamiltonian topology of $\underline{\mathbf{H}}$, its algebraic properties exhibit special features as it is shown in Benner, Mehrmann, and Xu (1998); Souriau (1997). Namely, it is known that if $\mu$ is an eigenvalue of $\underline{\underline{\mathbf{H}}}$, then $(-\mu)$ also belongs to its spectrum. Thus, the eigen-solutions of Equation (27) can be classified into these two categories:

$$
\mu_{i}^{+}=\mu_{i}, \quad \operatorname{Re}\left(\mu_{i}\right)>0 ; \quad \mu_{i}^{-}=-\mu_{i}
$$

The eigenvectors, classified according to Equation (30) and denoted as $\underline{\underline{\Psi}}_{2 n}=\left[\underline{\underline{\psi}}^{+}, \underline{\underline{\psi}}^{-}\right]$, satisfy the following symplectic-orthogonality relation:

$$
\underline{\underline{\Psi}}_{2 n}^{T} \underline{\underline{\mathbf{J}}} \underline{\underline{\Psi}}_{2 n}=\underline{\underline{\mathbf{J}}}
$$

Due to the fact that the eigenvectors $\left[\underline{\psi}^{+}, \underline{\psi}^{-}\right]$, solutions of Equation (27), form an orthonormal basis, thanks to Equation (31), the solution in Equation (26) can be written as a linear combination of these eigenvectors as follows:

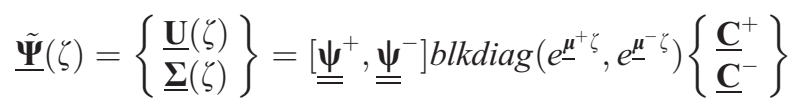

with blkdiag denoting a block diagonal matrix; later, diag is used for a diagonal one.

Hence, the integration of the original ODE (24) leads to the general solution for a singlelayer after substituting the previous (Equation (32)) result in Equation (19):

$$
\begin{aligned}
\hat{\underline{\Psi}} & =\left\{\begin{array}{l}
\underline{\mathrm{u}}(x, y, \zeta) \\
\underline{\underline{\sigma}}_{t}(x, y, \zeta)
\end{array}\right\}=\operatorname{blkdiag}(\underline{\underline{\mathrm{N}}}(x, y), \underline{\underline{\mathrm{N}}}(x, y))\left\{\begin{array}{l}
\underline{\mathrm{U}}(\zeta) \\
\underline{\underline{\Sigma}}(\zeta)
\end{array}\right\} \\
& =\operatorname{blkdiag}(\underline{\underline{\mathrm{N}}}, \underline{\underline{\mathrm{N}}})\left(\left[\underline{\underline{\Psi}}^{+}, \underline{\underline{\Psi}}^{-}\right] \text {blkdiag }\left(e^{\underline{\mu^{+}} \zeta}, e^{\underline{\underline{\mu}}} \zeta\right)\left\{\begin{array}{l}
\underline{\mathrm{C}}^{+} \\
\underline{\mathrm{C}}^{-}
\end{array}\right\}\right)
\end{aligned}
$$

where, $\underline{\mathbf{C}}=\left\{\underline{\mathbf{C}}^{+} \quad \underline{\mathbf{C}}^{-}\right\}^{T}$ is a constant vector to be determined, in the next sub-section, by the transverse stress $\mathrm{BC}$ on the plate bottom and top surfaces.

\subsection{State space propagator matrix - based laminate solution}

Following the discussion led in the preceding subsection, the $2 n \times 2 n$ eigenvector matrix $\underline{\underline{\Psi}}_{2 n}^{k}=\left[\begin{array}{ll}\underline{\underline{\Psi}}_{k}^{-} & \underline{\underline{\psi}}_{k}^{+}\end{array}\right]$for a given layer $k$, with the help of the symplectic-orthogonality relation stated in Equation (31), leads to this inversion formula: 


$$
\left(\underline{\underline{\Psi}}_{2 n}^{k}\right)^{-1}=-\underline{\underline{\mathbf{J}}} \underline{\underline{\Psi}}_{2 n}^{k T} \underline{\mathbf{J}}
$$

Now, letting $\zeta=0$ in the thickness solution (Equation (32)) of a single-layer gives this relation:

$$
\left\{\underline{\tilde{\boldsymbol{\Psi}}}^{k}\right\}_{\zeta=0}=\underline{\underline{\Psi}}_{2 n}^{\mathrm{k}} \underline{\mathbf{C}}^{k}
$$

Then, solving for the unknown constant vector $\underline{\mathbf{C}}^{k}=\left\{\underline{\mathbf{C}}_{k}^{+}, \underline{\mathbf{C}}_{k}^{-}\right\}^{T}$, by considering (34), gives:

$$
\underline{\mathbf{C}}^{k}=\left(\underline{\underline{\Psi}}_{2 n}^{k}\right)^{-1}\left\{\underline{\tilde{\boldsymbol{\Psi}}}^{k}\right\}_{\zeta=0}=-\underline{\underline{\mathbf{J}}}\left(\underline{\underline{\Psi}}_{2 n}^{k}\right)^{T} \underline{\underline{\mathbf{J}}}\left\{\underline{\tilde{\boldsymbol{\Psi}}}^{k}\right\}_{\zeta=0}
$$

Therefore, the thickness solution at a height $\zeta$ for a layer $k$ (Equation (32)) writes:

$$
\left\{\underline{\tilde{\boldsymbol{\Psi}}}^{k}\right\}_{\zeta}=\underline{\underline{\mathbf{p}}}_{k}(\zeta)\left\{\underline{\tilde{\boldsymbol{\Psi}}}^{k}\right\}_{\zeta=0}
$$

where, the propagator matrix $\underline{\underline{\mathbf{p}}}_{k}$, known also as the transfer matrix, is defined by:

$$
\underline{\underline{\mathbf{p}}}_{k}(\zeta)=-\underline{\underline{\Psi}}_{2 n}^{k} \operatorname{diag}\left(e^{\underline{\boldsymbol{\mu}}^{k}} \bar{\zeta}\right) \underline{\underline{\mathbf{J}}}\left(\underline{\underline{\Psi}}_{2 n}^{k}\right)^{T} \underline{\underline{\mathbf{J}}}
$$

with $\underline{\boldsymbol{\mu}}_{2 n}^{k}$ referring to eigenvalues of the Hamiltonian matrix relative to the layer $k$.

This transfer matrix can then be used recursively to compute the state vector $\left\{\tilde{\boldsymbol{\Psi}}^{k}\right\}_{\zeta}$ at any height $\zeta$ by satisfying the laminate IC. Namely, for an $N$-layered structure this relation holds:

$$
\left\{\underline{\tilde{\boldsymbol{\Psi}}}^{N}\right\}_{\zeta=h_{N}}=\underline{\underline{\mathbf{P}}}\left\{\underline{\tilde{\boldsymbol{\Psi}}}^{1}\right\}_{\zeta=0}
$$

where, $\left\{\tilde{\boldsymbol{\Psi}}^{j}\right\}_{\zeta=h_{j}}$ refers to the state vector of the $j$ th layer evaluated at $\zeta=h_{j}$ and

$$
\underline{\underline{\mathbf{P}}}=\prod_{k=N}^{k=1} \underline{\underline{p}}_{k}\left(h_{k}\right)
$$

The transverse stress $\mathrm{BC}$ on the top and bottom surfaces of the laminate can be inserted in Equation (39) in order to determine the unknown components of $\left\{\underline{\tilde{\boldsymbol{\Psi}}}^{1}\right\}_{\zeta=0}$ and $\left\{\tilde{\boldsymbol{\Psi}}^{k}\right\}_{\zeta}$ which are computed with the help of (35) and (37), respectively. Finally, the integration of the ODE (26) leads, with the help of Equations (19) and (37-38), to this general solution:

$$
\begin{aligned}
\left\{\begin{array}{l}
\underline{\mathbf{u}}^{k}\left(x_{j}, y_{j}, \zeta\right) \\
\underline{\boldsymbol{\sigma}}_{t}^{k}\left(x_{j}, y_{j}, \zeta\right)
\end{array}\right\} & =\text { blkdiag }\left[\underline{\underline{\mathbf{N}}}\left(x_{j}, y_{j}\right), \underline{\underline{\mathbf{N}}}\left(x_{j}, y_{j}\right)\right]\left\{\begin{array}{l}
\underline{\underline{\mathbf{U}}}^{k}(\zeta) \\
\underline{\underline{\boldsymbol{\Sigma}}}_{t}^{k}(\zeta)
\end{array}\right\} \\
& =\text { blkiag }\left[\underline{\underline{\mathbf{N}}}\left(x_{j}, y_{j}\right), \underline{\underline{\mathbf{N}}}\left(x_{j}, y_{j}\right)\right] \underline{\underline{\mathbf{P}}}^{k}(\zeta)\left\{\underline{\tilde{\boldsymbol{\Psi}}}^{1}\right\}_{\zeta=0}
\end{aligned}
$$

where,

$$
\underline{\underline{\mathbf{P}}}^{k}(\zeta)=\underline{\underline{\mathbf{p}}}_{k}(\zeta) \prod_{l=(k-1)}^{l=1} \underline{\underline{\mathbf{p}}}_{l}\left(h_{l}\right)
$$

And $\left(x_{j}, y_{j}\right)$ are the locations of nodal points on the reference plane (here at $z=0$ ). 


\subsection{Implementation}

The present semi-analytical partial mixed FE-SSM Hamiltonian approach has been imple-

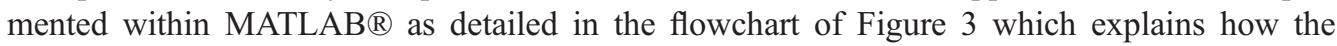
code is organised and its computations sequences order.

It is worth noting that the procedure outlined here allows solving a system in which size is independent of the laminate number of layers; hence, this partial-mixed special FE computational cost can be reduced to a reasonable level. Actually, the cost of the computations mainly relates to that of the in-plane FE procedure.

\section{Analysis of multilayer composites and FGM}

In order to demonstrate the performance and accuracy of the present semi-analytical FE-SSM Hamiltonian approach, a standard linear four nodes quadrangular (Q4) plate FE, having the 3 translations and 3 transverse stresses as degrees of freedom (DOF), has been implemented in MATLAB ${ }^{\circledR}$ and used for the static analysis of selected multilayer composite and FGM SS plate benchmarks available from the open literature. Throughout all the analysed examples, these dimensionless notations are used for the primary and secondary output variables, respectively:

$$
\begin{gathered}
\bar{u}=\frac{100 E_{T}}{F^{o} h S^{3}} u, \bar{w}=\frac{100 E_{T}}{F^{o} h S^{4}} w, \sigma_{x z}^{*}=\frac{1}{F^{\circ}} \sigma_{x z}, \sigma_{z z}^{*}=\frac{1}{F^{\circ}} \sigma_{z z} \\
\sigma_{x x}^{*}=\frac{1}{F^{\circ} S^{2}} \sigma_{x x}, \sigma_{x y}^{*}=\frac{1}{F^{\circ} S^{2}} \sigma_{x y}, S=\frac{a}{h}
\end{gathered}
$$

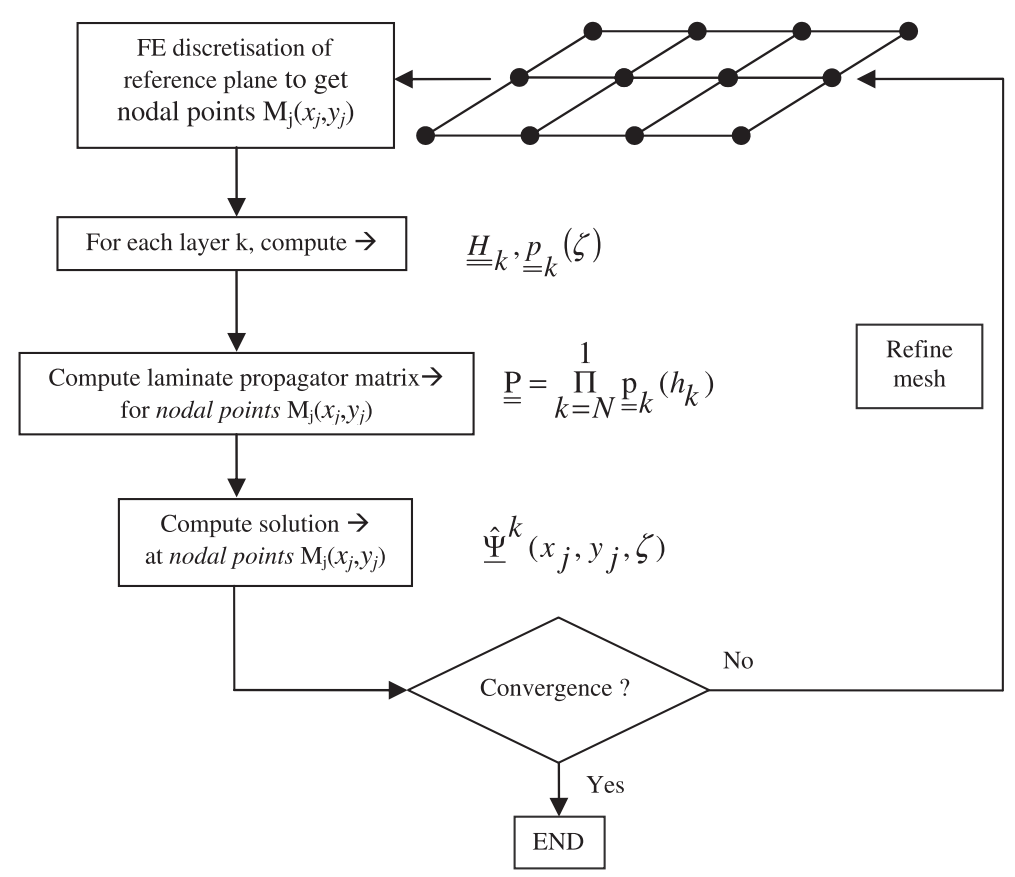

Figure 3. FE-SSM semi-analytical approach computations sequences order. 
where, $F^{\circ}$ is the load amplitude, $a$ (resp. $h$ ) is the plate length (resp. thickness) and $E_{T}$ refers to the Young modulus relative to the direction transverse to the fibres.

\subsection{Cross-ply bi-layer square plate}

This first example deals with the analysis of a SS bi-layer composite square $(a=b=1 \mathrm{~m})$ and moderately thick $(S=10)$ plate (Auricchio \& Sacco, 1999). It is subjected to a uniformly distributed load $F=1 \mathrm{~N} / \mathrm{m}^{2}$ on the top surface and has a $\left[0^{\circ} / 90^{\circ}\right]$ layup. The total thickness of the plate is $h$ and both layers of the laminate are taken to be of the same thickness and orthotropic fibre-reinforced composite (FRC) material which properties in the material coordinates system are: $\mathrm{C}_{11}=173.527 \mathrm{GPa}, \mathrm{C}_{12}=\mathrm{C}_{13}=2.314 \mathrm{GPa}, \mathrm{C}_{22}=\mathrm{C}_{33}=7.385 \mathrm{GPa}, \mathrm{C}_{23}=1.869$ $\mathrm{GPa}, \mathrm{C}_{44}=1.379 \mathrm{GPa}$ and $\mathrm{C}_{55}=\mathrm{C}_{66}=3.447 \mathrm{GPa}$.

Table 1 presents the convergence analysis of the present semi-analytical solution with regard to the reference analytical solution from Auricchio and Sacco (1999); results by a full mixed state-space FE (Sheng \& Ye, 2002) are also given for comparison purpose. It can be seen from Table 1 that the results obtained using the present Q4 partial-mixed special FE are in good accordance with the exact solutions for a mesh size of $8 \times 8$ elements. However, the convergence is much lower than Sheng and Ye (2002) probably due to used higher FE approximations; this information was absent in the latter reference. In view of this convergence analysis, an $8 \times 8 \mathrm{FE}$ mesh will be then used for the subsequent benchmarking examples.

Figures 4-6, showing the through-thickness distributions of some variables through the plate thickness, indicate that the primary variables (displacement and transverse stresses) IC and top and bottom surfaces transverse stresses BC are satisfied; while, as expected, the secondary variables (in-plane stresses) may be discontinuous at the plate interface and the inplane and transverse displacements can be seen (cf. horizontal scale values) thickness linear and constant, respectively.

\subsection{Cross-ply three-layered rectangular plate}

A SS rectangular $\left[0^{\circ} / 90^{\circ} / 0^{\circ}\right]$ laminate of length $a$ in the $x$ direction and $b=3 a$ in the $y$ direction is now subjected to a bi-sinusoidal pressure of amplitude $1 \mathrm{~N} \mathrm{~m}^{-2}$ on its top surface. The obtained dimensionless deflection and transverse stresses are presented in Table 2. Exact 3D (Pagano, 1970) solutions are also reported for comparison and Figures 7-9 show the throughthickness distribution of these variables. Results show that the present solutions are in good

Table 1. Convergence analysis of a moderately thick $(S=10)$ SS square $(a=b=1 \mathrm{~m})\left[0^{\circ} / 90^{\circ}\right]$ plate under a uniform load $\left(F=1 \mathrm{~N} / \mathrm{m}^{2}\right)$.

\begin{tabular}{lccccc}
\hline Auricchio and Sacco (1999) & In-plane Mesh & Present & Error* $\left.^{*} \%\right)$ & Sheng and Ye (2002) & Error* (\%) \\
\hline $\bar{u}(0, \mathrm{a} / 2, \mathrm{~h} / 2)(-\mathbf{0 . 1 2 9 4})$ & $2 \times 2$ & -0.0786 & -39.26 & -0.1298 & 0.31 \\
& $4 \times 4$ & -0.1073 & -17.08 & -0.1301 & 0.54 \\
& $6 \times 6$ & -0.1215 & -6.11 & -0.1301 & 0.54 \\
& $8 \times 8$ & -0.1281 & -1.00 & $\mathrm{NA}$ & $\mathrm{NA}$ \\
$\bar{w}(\mathrm{a} / 2, \mathrm{a} / 2, \mathrm{~h} / 2)(\mathbf{1 . 9 4 6 9})$ & $2 \times 2$ & 1.3501 & -30.65 & 1.9379 & -0.46 \\
& $4 \times 4$ & 1.6723 & -14.10 & 1.9380 & -0.46 \\
& $6 \times 6$ & 1.8610 & -4.41 & 1.9381 & -0.45 \\
& $8 \times 8$ & 1.9320 & -0.77 & $\mathrm{NA}$ & $\mathrm{NA}$ \\
\hline
\end{tabular}

Note: Error* (\%) measures relative deviation between numerical and analytical solution (in boldface) as in Auricchio and Sacco (1999); NA abbreviates not available. 
accordance with 3D solutions for thick $(S=4)$, moderately thick $(S=10)$ and thin $(S=50)$ plates.

\subsection{Angle-ply bi-layer square plate}

Analysis of laminated composite plates where the principal material directions do not coincide with the axes of geometry constitutes a challenging test case. Namely, it is well known that
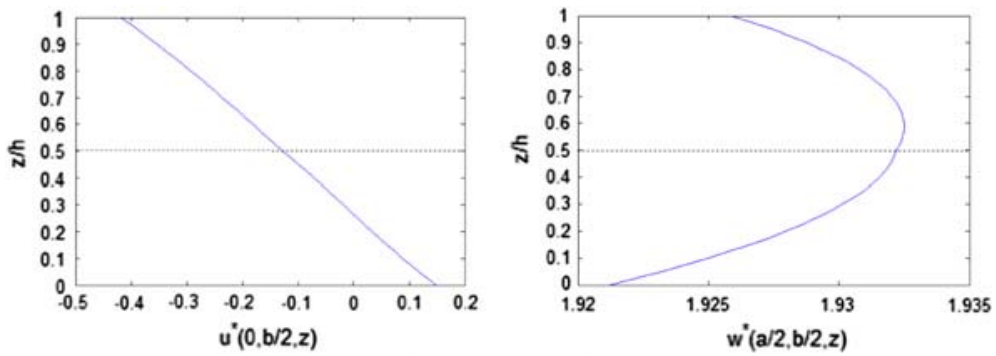

Figure 4. Dimensionless displacements thickness profiles for a moderately thick $(S=10)$ SS square $(a=b=1 \mathrm{~m})\left[0^{\circ} / 90^{\circ}\right]$ plate under a uniform load $\left(F=1 \mathrm{~N} / \mathrm{m}^{2}\right)$.
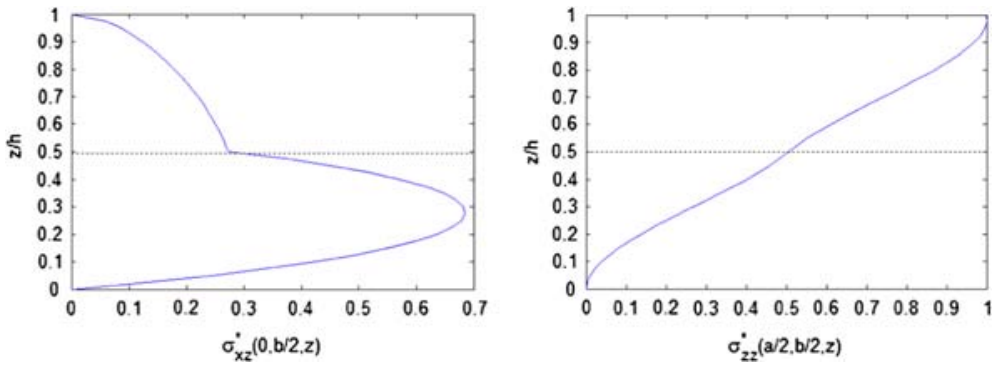

Figure 5. Dimensionless transverse stresses thickness profiles for moderately thick $(S=10)$ SS square $(a=b=1 \mathrm{~m})\left[0^{\circ} / 90^{\circ}\right]$ plate under uniform load $\left(F=1 \mathrm{~N} / \mathrm{m}^{2}\right)$.
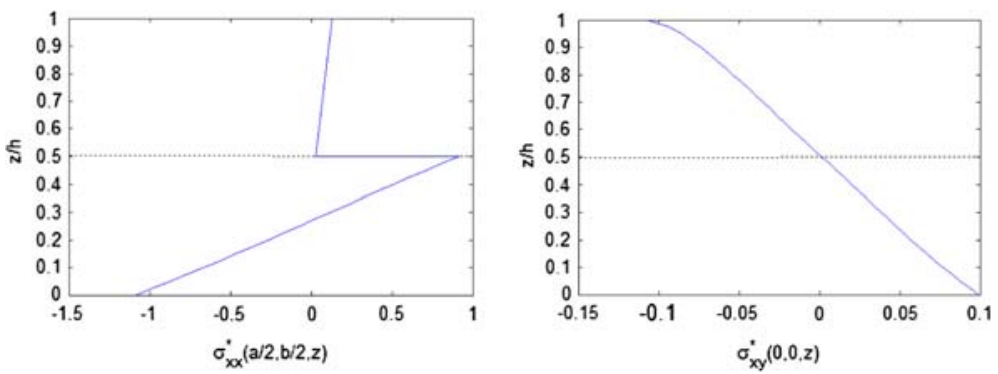

Figure 6. Dimensionless in-plane stresses thickness profiles for a moderately thick $(S=10)$ SS square $(a=b=1 \mathrm{~m})\left[0^{\circ} / 90^{\circ}\right]$ plate under a uniform load $\left(F=1 \mathrm{~N} / \mathrm{m}^{2}\right)$. 
such angle-ply composite plates are characterised by specific response coupling effects due to nonvanishing bending-twisting coupling stiffness terms $C_{i 6}(i=1,2,3)$ or shear-extensional coupling constant $C_{45}$; their consideration substantially increases the complexity of the analysis and makes it more complex than that of cross-ply plates as in the first benchmark.

The test case considered here consists of a SS square $(a=b=1 \mathrm{~m})$ plate with two layers of equal thickness and ply orientations $\left[\theta^{\circ} /-\theta^{\circ}\right]$. It is subjected to transverse uniform $F=1 \mathrm{~N} /$ $\mathrm{m}^{2}$ or bi-sinusoidal load with amplitude $F^{\circ}=1 \mathrm{~N} / \mathrm{m}^{2}$. The layers elastic properties are (Desai, Ramtekkar, \& Shah, 2003): $E_{1}=276 \mathrm{GPa}, E_{2}=E_{3}=6.89 \mathrm{GPa}, \quad G_{12}=G_{13}=3.45 \mathrm{GPa}$, $G_{23}=4.2 \mathrm{GPa}, v_{12}=v_{13}=v_{23}=0.25$. Obtained results, reported in Table 3 for the plate interface and centre normalised transverse displacement, show reasonable correlation with the 3D mixed FE results presented in Desai, Ramtekkar, and Shah (2003).

Figures 10-12 illustrate the results presented in Table 3 for $\theta=15^{\circ}$ and the transverse and in-plane stresses thickness profiles for $S=4,10,100$ under bi-sinusoidal pressure. Beside the primary variables IC and stress load BC satisfaction, it can be observed that the stresses increase with increasing the span ratio; besides, the in-plane displacements and stresses become nonlinear for the thick case $(S=4)$.

\subsection{FGM bi-layer square plate}

This last example deals with the static bending analysis of a SS bi-layer square $(a=b=1 \mathrm{~m})$ plate composed of FGM and orthotropic layers stacked as [0\% FGM] (Figure 13) and under this bi-sinusoidal distributed load of amplitude $F^{\circ}=1 \mathrm{~N} / \mathrm{m}^{2}$ :

$$
F=F^{\circ} \sin \left(\frac{\pi x}{a}\right) \sin \left(\frac{\pi y}{b}\right)
$$

The two layers are of equal thickness and the material properties are graduated as in $\mathrm{Wu}$ and Li (2010b) such that (denotes the property grading index):

Table 2. Dimensionless transverse displacement and stresses for a 3-layered SS rectangular plate under bi-sinusoidal load of amplitude $F=1 \mathrm{Nm}^{-2}$.

\begin{tabular}{lrclr}
\hline Variable & $S$ & (Pagano, 1970) & Present & Error* (\%) \\
\hline $\bar{w}(a / 2, b / 2, h / 2)$ & 4 & 2.82 & 2.831 & 0.39 \\
& 10 & 0.919 & 0.9169 & -0.23 \\
& 50 & 0.520 & 0.5157 & -0.82 \\
$\sigma_{x z}^{*}(0, b / 2, h / 2)$ & 4 & 0.351 & 0.352 & 0.28 \\
& 10 & 0.420 & 0.4218 & 0.43 \\
& 50 & 0.439 & 0.4409 & 0.43 \\
$\sigma_{y z}^{*}(a / 2,0, h / 2)$ & 4 & 0.0334 & & 0.92 \\
& 10 & 0.0152 & $0.03,371$ & -0.85 \\
& 50 & 0.011 & $0.01,507$ & -1.82 \\
$\sigma_{z z}^{*}(a / 2, b / 2, h / 2)$ & 4 & & & NA \\
& 40 & NA & 0.5 & NA \\
& 50 & NA & 0.5 & NA \\
\hline
\end{tabular}

Note: Error ${ }^{*}(\%)$ measures relative deviation between numerical and analytical solution (Pagano, 1970); NA abbreviates not available. 

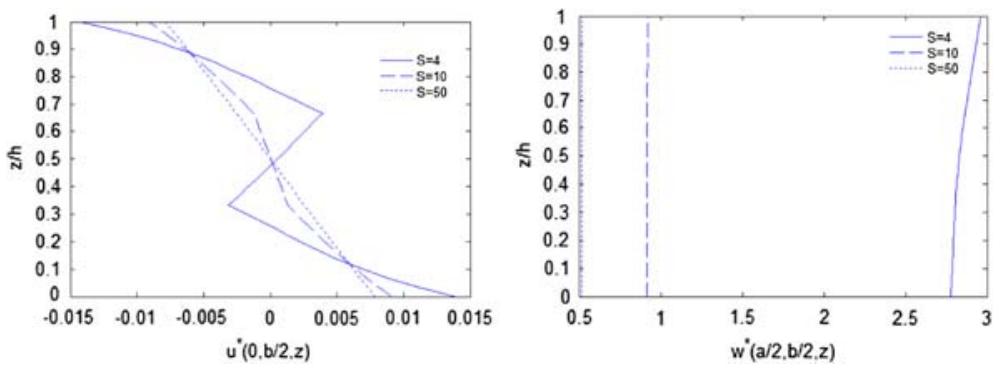

Figure 7. Dimensionless displacement thickness profiles for varying ratio S. SS rectangular $(b=3 a)$ $\left[0^{\circ} / 90^{\circ} / 0^{\circ}\right]$ plate under a bi-sinusoidal load $\left(F^{\circ}=1 \mathrm{~N} / \mathrm{m}^{2}\right)$.
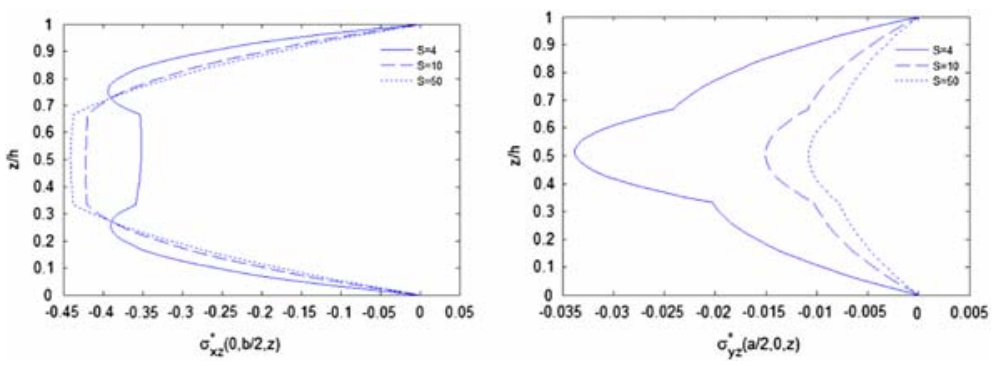

Figure 8. Dimensionless transverse stresses thickness profiles for varying ratio S. SS rectangular $(b=3 a)\left[0^{\circ} / 90^{\circ} / 0^{\circ}\right]$ plate under a bi-sinusoidal load $\left(F^{\circ}=1 \mathrm{~N} / \mathrm{m}^{2}\right)$.
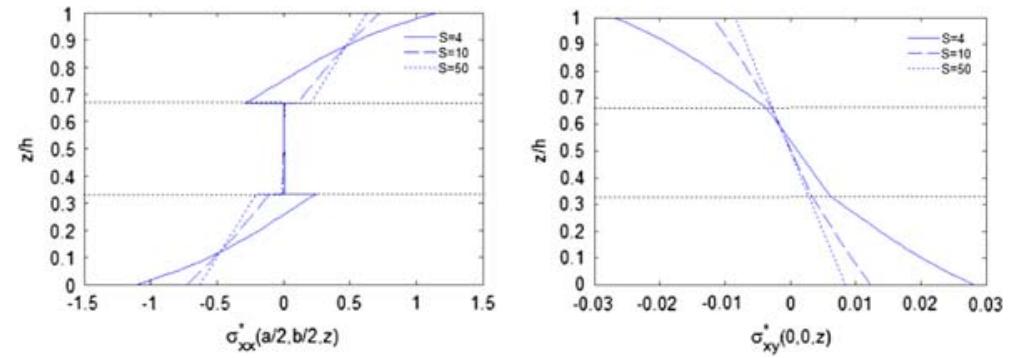

Figure 9. Dimensionless in-plane stresses thickness profiles for varying ratio S. SS rectangular $(b=3 a)$ $\left[0^{\circ} / 90^{\circ} / 0^{\circ}\right]$ plate under a bi-sinusoidal load $\left(F^{\circ}=1 \mathrm{~N} / \mathrm{m}^{2}\right)$.

$$
C_{i j}=C_{i j}^{o}, 0 \leq z \leq H_{1} ; \quad C_{i j}=C_{i j}^{o} e^{\eta_{h}^{z}}, H_{1} \leq z \leq H_{2}
$$

For a general thickness distribution pattern of the material properties, characterised by a continuous function $F(z)$, the problem can be solved by dividing the laminate into thin sublayers, each one having piezoelectric constants defined as:

$$
\underline{\underline{\mathbf{C}}}^{k}=\underline{\underline{\mathbf{C}}}_{b}^{k}(1-\zeta)+\zeta \underline{\underline{\mathbf{C}}}_{t}^{k} ; \underline{\underline{\mathbf{C}}}_{b}^{k}=\underline{\underline{\mathbf{C}}}^{o} F\left(z_{b}\right), \underline{\underline{\mathbf{C}}}_{t}^{k}=\underline{\underline{\mathbf{C}}}^{o} F\left(z_{t}\right)
$$


Table 3. Dimensionless transverse displacement at the centre and interface for varying span-tothickness ratio of SS square $(a=b=1 \mathrm{~m})[\theta /-\theta]$ plate under a bi-sinusoidal pressure or uniform load.

\begin{tabular}{|c|c|c|c|c|c|c|c|}
\hline \multirow[b]{2}{*}{$S$} & \multirow{2}{*}{$\begin{array}{l}\text { Load } \\
\theta\end{array}$} & \multicolumn{3}{|c|}{ Bi-sinusoïdal } & \multicolumn{3}{|c|}{ Uniform } \\
\hline & & $15^{\circ}$ & $30^{\circ}$ & $45^{\circ}$ & $15^{\circ}$ & $30^{\circ}$ & $45^{\circ}$ \\
\hline \multirow[t]{3}{*}{4} & Present & 1.467 & 1.455 & 1.418 & 2.409 & 2.391 & 2.330 \\
\hline & (Desai, Ramtekkar, \& Shah, 2003) & 1.548 & 1.519 & 1.515 & 2.276 & 2.361 & 2.356 \\
\hline & Error* $^{*}(\%)$ & -5.23 & -4.21 & -6.40 & 5.84 & 1.27 & -1.10 \\
\hline \multirow[t]{3}{*}{10} & Present & 0.644 & 0.669 & 0.638 & 1.056 & 1.096 & 1.046 \\
\hline & (Desai, Ramtekkar, \& Shah, 2003) & 0.663 & 0.731 & 0.723 & 1.021 & 1.141 & 1.131 \\
\hline & Error* $^{*}(\%)$ & -2.87 & -8.48 & -11.76 & -3.43 & -3.94 & -7.52 \\
\hline \multirow[t]{3}{*}{100} & Present & 0.468 & 0.497 & 0.468 & 0.764 & 0.809 & 0.762 \\
\hline & (Desai, Ramtekkar, \& Shah, 2003) & 0.459 & 0.488 & 0.463 & 0.708 & 0.773 & 0.735 \\
\hline & Error* $(\%)$ & 1.96 & 1.84 & 1.08 & 7.91 & 4.66 & 3.67 \\
\hline
\end{tabular}

Note: Error* (\%) measures relative deviation between numerical and 3D mixed FE solution as in Desai, Ramtekkar, and Shah (2003).
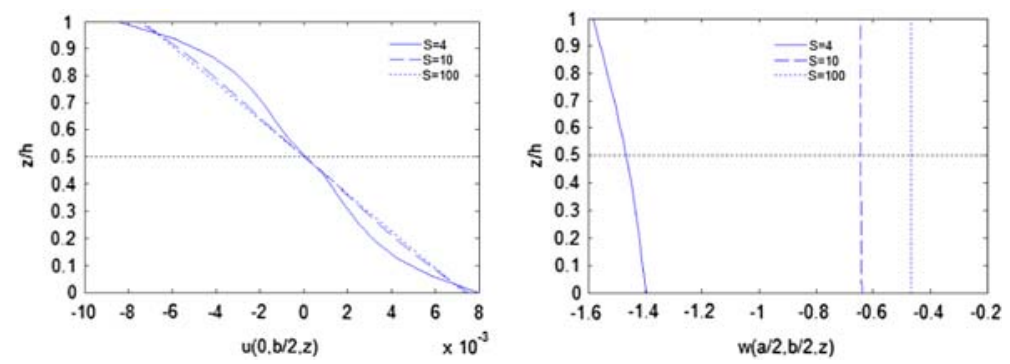

Figure 10. Dimensionless displacements thickness profiles for varying span-to-thickness ratio of a SS square $(a=b=1 \mathrm{~m})\left[15^{\circ} /-15^{\circ}\right]$ plate under bi-sinusoidal transverse distributed load with amplitude $F^{\circ}$ $=1 \mathrm{~N} / \mathrm{m}^{2}$.
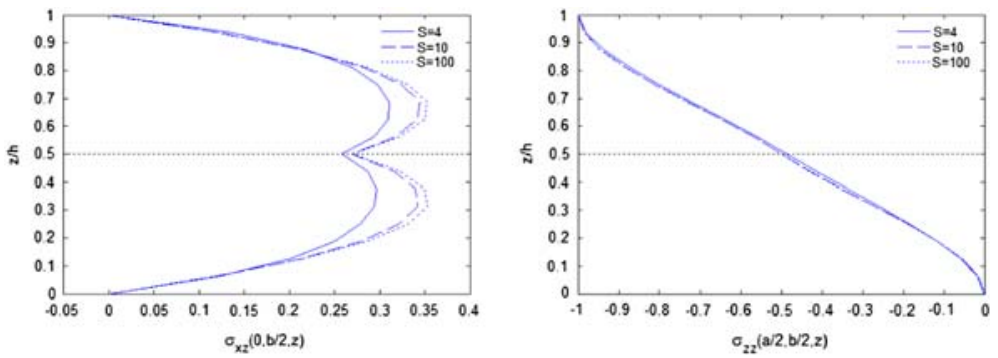

Figure 11. Dimensionless transverse stresses thickness profiles for varying span-to-thickness ratio of a SS square $(a=b=1 \mathrm{~m})\left[15^{\circ} /-15^{\circ}\right]$ plate under bi-sinusoidal transverse distributed load with amplitude $F$ $\circ=1 \mathrm{~N} / \mathrm{m}^{2}$.

where, $z_{b}, z_{t}$ are the global coordinates of the bottom and top of the $k$ th sub-layer with local coordinate $\zeta$. Equation (45) is obviously true only if the sub-layers are sufficiently thin so that the properties can be assumed to be linearly distributed through these thin plies. $C_{i j}^{0}$ are those given in Example 1 (cross-ply bi-layer plate). 

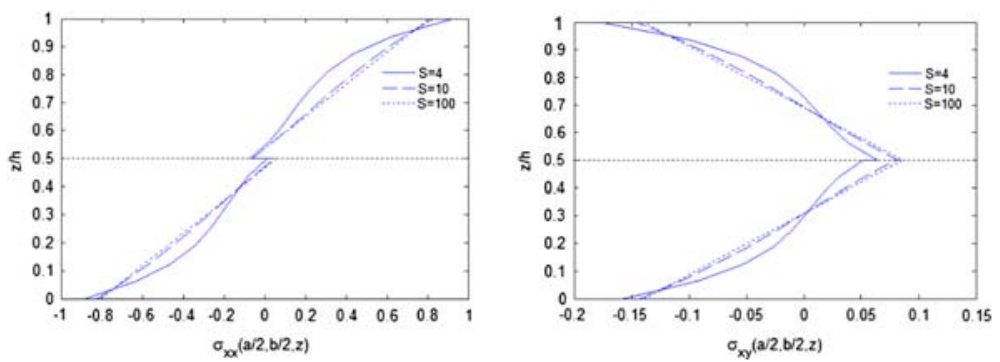

Figure 12. Dimensionless in-plane stresses thickness profiles for varying span-to-thickness ratio of a SS square $(a=b=1 \mathrm{~m})\left[15^{\circ} /-15^{\circ}\right]$ plate under bi-sinusoidal transverse distributed load with amplitude $F$ $\circ=1 \mathrm{~N} / \mathrm{m}^{2}$.

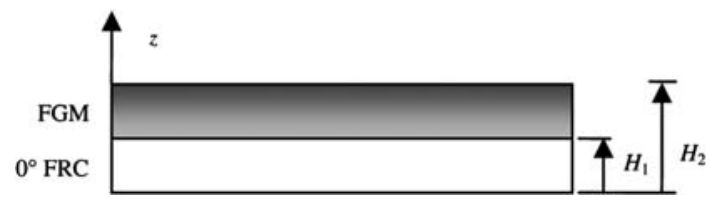

Figure 13. FGM bi-layer configuration.

Tables 4 and 5 present in-plane and transverse shear stresses results at some selected points for aspect ratio $S=5,10,50$ and grading index $\eta=3,5$, respectively; obviously, it was first checked that the homogeneous $(\eta=0)$ plate solution is recovered. Numerical layers $(8-$ 10) are used to model the FGM layer. Comparison with the exact solution, given in Wu and Li (2010b), shows for both grading index values better correlation than the latter reference results, obtained using a third-order shear deformation theory (TSDT)-based plate partialmixed $\mathrm{FE}$, for the thick $(S=5)$ and moderately thick $(S=10)$ cases and their transverse shear stress values. These benefits to the present partial-mixed special FE, may come from the thickness analytical integration and the transverse normal stress effect that was dropped by $\mathrm{Wu}$ and $\mathrm{Li}$ (2010b). The results of both solutions are similar for the thin $(S=100)$ case indicating that the present low-order (Q4) partial-mixed special FE recovers this degenerating case.

Figure 14-16 show thickness profiles of dimensionless primary and secondary output variables for varying gradient index $\eta$ and span ratio $S=10$. It is observed that out-of-plane displacement values decrease when the values of $\eta$ increase. It is also seen that the maximum value of the transverse shear stress, $\sigma_{x z}$ (evaluated at $\mathrm{z}=h / 2$ ), is an increasing function of $\eta$. Moreover the $z$-location of the point where $\sigma_{x z}$ is maximum tends to move into the FGM part of the plate. This means that the latter tends to withstand an increasing part of the load with augmenting the gradient index.

\section{Conclusions and perspectives}

A partial-mixed special FE for the semi-analytical analysis of multilayer composite and FGM plates was presented within the framework of the Hamiltonian formalism that led to a variational formulation in terms of displacements and transverse stresses only as independent variables. After an in-plane FE discretisation only, a Hamiltonian eigenvalue problem was 
Table 4. Dimensionless stresses for varying span-to-thickness ratio of a SS square $(a=b=1 \mathrm{~m})\left[0^{\circ} /\right.$ FGM] $(\eta=3)$ plate under bi-sinusoidal pressure of $F^{\circ}=1 \mathrm{~N} / \mathrm{m}^{2}$.

\begin{tabular}{lrlcccr}
\hline Variable & \multicolumn{1}{c}{$S$} & Exact & Present & Error $^{*}(\%)$ & Wu and Li (2010b) & Error* (\%) \\
\hline$\sigma_{x x}^{*}(a / 2, a / 2, h)$ & 5 & 3.1521 & 3.1947 & 1.35 & 2.8587 & -9.31 \\
& 10 & 2.2490 & 2.280 & 1.38 & 2.1268 & -5.43 \\
& 100 & 1.8478 & 1.8732 & 1.37 & 1.8713 & 1.27 \\
$\sigma_{x z}^{*}(0, a / 2, h / 2)$ & 5 & 0.2570 & 0.2563 & -0.27 & & 0.2867 \\
& 10 & 0.3237 & 0.3230 & -0.22 & 0.3375 & 11.56 \\
& 100 & 0.3586 & 0.3580 & -0.17 & 0.3582 & -0.11 \\
$\sigma_{x y}^{*}(0,0, h)$ & 5 & -0.1480 & -0.1497 & 1.15 & -0.1459 & -1.42 \\
& 10 & -0.0970 & -0.0983 & 1.34 & -0.0951 & -1.96 \\
& 100 & -0.0731 & -0.0741 & 1.37 & -0.0741 & 1.37 \\
\hline
\end{tabular}

Note: Error* (\%) measures relative deviation between numerical and exact solution as in Wu and Li (2010b).

Table 5. Dimensionless stresses for varying span-to-thickness ratio of a SS square $(a=b=1 \mathrm{~m})\left[0^{\circ}\right.$ FGM] $(\eta=5)$ plate under bi-sinusoidal pressure of $F^{\circ}=1 \mathrm{~N} / \mathrm{m}^{2}$.

\begin{tabular}{|c|c|c|c|c|c|c|}
\hline Variable & $S$ & Exact & Present & Error* $(\%)$ & $\mathrm{Wu}$ and $\mathrm{Li}(2010 \mathrm{~b})$ & Error* $(\%)$ \\
\hline \multirow{3}{*}{$\sigma_{x x}^{*}(a / 2, a / 2, \mathrm{~h})$} & 5 & 7.9053 & 8.0686 & 2.07 & 7.3856 & -6.57 \\
\hline & 10 & 5.7358 & 5.8666 & 2.28 & 5.3838 & -6.14 \\
\hline & 100 & 4.5429 & 4.6530 & 2.42 & 4.6459 & 2.27 \\
\hline \multirow[t]{3}{*}{$\sigma_{x z}^{*}(0, a / 2, h / 2)$} & 5 & 0.1516 & 0.1504 & -0.79 & 0.1986 & 31.0 \\
\hline & 10 & 0.2260 & 0.2244 & -0.71 & 0.2519 & 11.46 \\
\hline & 100 & 0.2742 & 0.2726 & -0.58 & 0.2730 & -0.44 \\
\hline \multirow[t]{3}{*}{$\sigma_{x y}^{*}(0,0, h)$} & 5 & -0.3394 & -0.3461 & 1.97 & -0.3416 & 0.65 \\
\hline & 10 & -0.2361 & -0.2412 & 2.16 & -0.2291 & -2.96 \\
\hline & 100 & -0.1797 & -0.1839 & 2.34 & -0.1838 & 2.28 \\
\hline
\end{tabular}

Note: Error ${ }^{*}(\%)$ measures relative deviation between numerical and exact solution as in Wu and Li (2010b).

obtained and the form of the analytical general solution was derived for a single layer with the help of the symplectic nature of the Hamiltonian matrix eigenvectors. Extension to the multilayer was then achieved using the analytical state space-method (SSM) combined with the related propagator matrix concept.
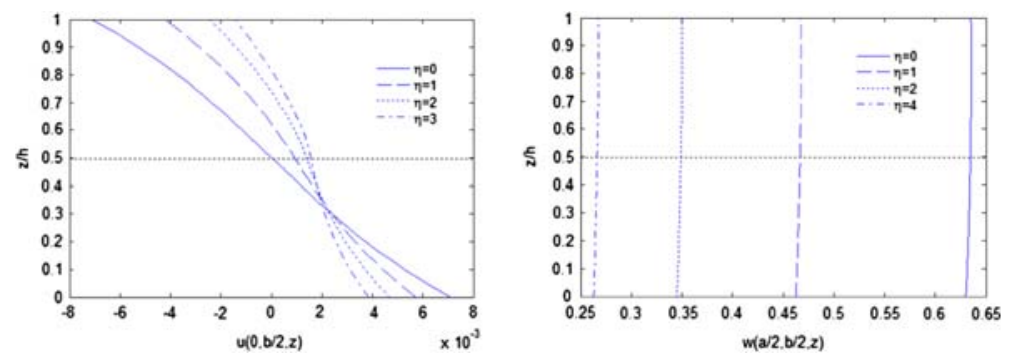

Figure 14. Dimensionless displacements for varying $\eta$ of moderately thick $(S=10)$ SS square $(a=b=1 \mathrm{~m})\left[0^{\circ} / \mathrm{FGM}\right]$ plate under bi-sinusoidal pressure $\left(F^{\circ}=1 \mathrm{~N} / \mathrm{m}^{2}\right)$. 

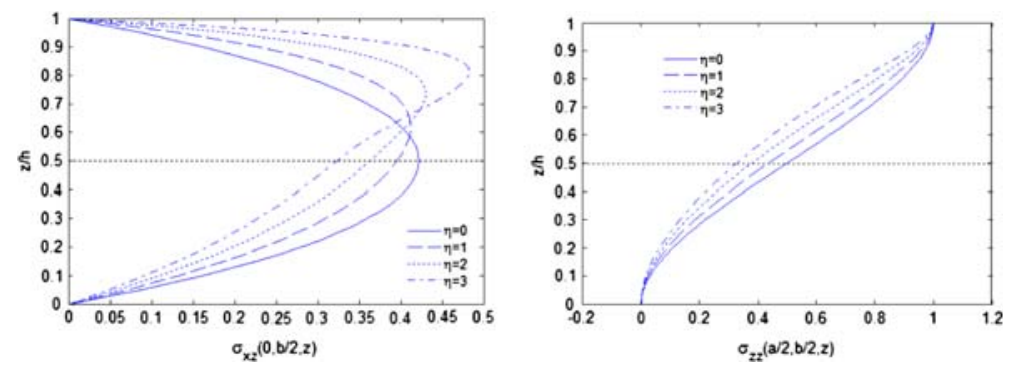

Figure 15. Dimensionless transverse stresses for varying grading index of a moderately thick $(S=10)$ SS square $(a=b=1 \mathrm{~m})\left[0^{\circ} / \mathrm{FGM}\right]$ plate under bi-sinusoidal pressure of $F^{\circ}=1 \mathrm{~N} / \mathrm{m}^{2}$.
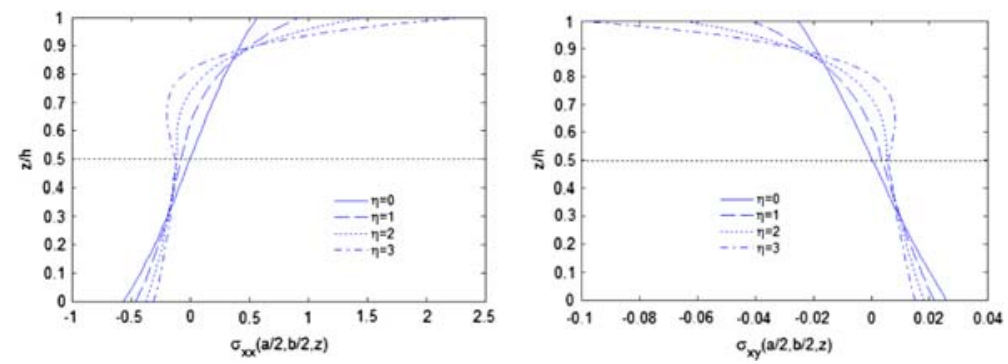

Figure 16. Dimensionless in-plane stresses for varying grading index of a moderately thick $(S=10) \mathrm{SS}$ square $(a=b=1 \mathrm{~m})\left[0^{\circ} / \mathrm{FGM}\right]$ plate under bi-sinusoidal pressure of $F^{\circ}=1 \mathrm{~N} / \mathrm{m}^{2}$.

The computational performance of the proposed low-order four nodes quadrangular partial-mixed special FE was assessed through the convergence and accuracy analysis of its primary and secondary output variables resulting from the static bending analysis of representative multilayer composite and FGM plates from the open literature. Satisfactory behaviour was observed for all run benchmarks.

This work has been already extended to piezoelectric multilayer composite and FGM plates; corresponding electromechanically coupled semi-analytical partial-mixed special FESSM Hamiltonian approach and results have been presented elsewhere (Andrianarison and Benjeddou, 2012). The next step will be to extend the present elastic and companion works to these advanced structures modal analysis.

\section{Acknowledgement}

Support of the second author, as international scientific partner, from the COMET K2-Austrian Center of Competence in Mechatronics (ACCM) at Linz is gratefully acknowledged.

\section{References}

Andrianarison, O., \& Benjeddou, A. (2012). Hamiltonian partial mixed finite element - state space symplectic semi-analytical approach for the piezoelectric composites and FGM analysis. Acta Mechanica. doi:10.1007/s00707-012-0646-8.

Auricchio, F., \& Sacco, E. (1999). A mixed-enhanced finite element for the analysis of laminated composite plates. International Journal for Numerical Methods in Engineering, 44, 1481-1504.

Bahar, L.Y. (1977). A mixed method in elasticity. Journal of Applied Mechanics, 44, 790-791. 
Benjeddou, A., \& Andrianarison, O. (2006). A heat mixed variational theorem for thermoelastic multilayered composites. Computers and Structures, 84(19-20), 1247-1255.

Benner, P., Mehrmann, V., \& Xu, H. (1998). A numerically stable, structure preserving method for computing the eigenvalues of real Hamiltonian or symplectic pencils. Numerische Matematik, 78, 32293258.

Birman, V., \& Byrd, L.W. (2007). Modeling and analysis of functionally graded materials and structures. Applied Mechanics Reviews, 60(5), 195-216.

Brezzi, F., \& Fortin, M. (1991). Mixed and hybrid finite element method. Springer-Verlag.

Courant, R., \& Hilbert, D. (1989). Methods of mathematical physics I, II. USA: John Wiley.

Desai, Y.M., Ramtekkar, G.S., \& Shah, A.H. (2003). A novel 3D mixed finite element model for statics of angle-ply laminates. International Journal for Numerical Methods in Engineering, 57, 16951716.

Goldstein, H., Poole, C., \& Safko, J. (2002). Classical mechanics. London: Addison Wesley.

Kreja, I. (2011). A literature review on computational models for laminated composite and sandwich panels. Central European Journal of Engineering, 1(1), 59-80.

Li, R., Zhong, Y., \& Tian, B. (2011). On new symplectic superposition method for exact solutions of rectangular cantilever thin plates. Mechanics Research Communications, 38, 111-116.

Pagano, N.J. (1970). Exact solutions for rectangular bidirectional composites and sandwich plates. Journal of Composite Materials, 4, 20-34.

Reissner, E. (1984). On a certain mixed variational theory and proposed applications. International Journal of Numerical Methods in Engineering, 20, 1366-1368.

Robaldo, A., Benjeddou, A., \& Carrera, E. (2005). Unified formulation for FE thermoelastic analysis of multilayered anisotropic composite plates. Journal of Thermal Stresses, 28(10), 1031-1065.

Sheng, H.Y., \& Ye, J.Q. (2002). A state space finite element for laminated composite plates. Computer Methods in Applied Mechanics and Engineering, 191, 4259-4276.

Sheng, H.Y., \& Ye, J.Q. (2005). A state space finite element for axisymmetric bending of angle-ply laminated cylinder with clamped edges. Composite Structures, 68, 119-128.

Souriau J.M. (1997). Structures of dynamical systems: A symplectic view of physics. Progress in Mathematics 149. Paris: Lavoisier.

Steele, C.R., \& Kim, Y.Y. (1992). Modified mixed variational principle and the state-vector equation for elastic bodies and shells of revolution. Journal of Applied Mechanics, 59, 587-595.

Van Loan, C. (1984). A symplectic method for approximating all the eigenvalues of a Hamiltonian matrix. Linear Algebra Application, 61, 233-251.

Washizu, K. (1968). Variational method in elasticity and plasticity. New York, NY: Pergamon Press.

Wu, C.P., \& Li, H.Y. (2010a). The RMVT and PVD based finite layer methods for the threedimensional analysis of multilayered composite and FGM plates. Composite Structures, 92, 24762496.

Wu, C.P., \& Li, H.Y. (2010b). An RMVT-based third order shear deformation theory for multilayered functionally graded material plate. Composite Structures, 92, 2591-2595.

Zhang, Y.X., \& Yang, C.H. (2009). Recent developments in finite element analysis for laminated composite plates. Composite Structures, 88, 147-157.

Zou, G., \& Tang, L. (1995). A semi-analytical solution for laminated composite plates in Hamilltonian system. Computer Methods in Applied Mechanics and Engineering, 128, 395-404. 